\title{
Preserved cerebral function after resuscitation and induction of hypothermia with packed snow ${ }^{1}$
}

\author{
Alexander J. Pfister ${ }^{a}$, Patrik R. Schwab ${ }^{a}$, Andreas Wahl ${ }^{b}$,Jukka Takala ${ }^{\text {, Bernhard Meier }}{ }^{b}$, Carlo Höfliger ${ }^{a}$ \\ a Swiss Air Rescue REGA, REGA Centre, Zurich Airport, Zurich, Switzerland \\ b Cardiology, Swiss Cardiovascular Centre Bern, University of Bern, Bern, Switzerland \\ c Department of Intensive Care, Emergency Medicine and Anaesthesiology, Intensive Care Clinic, \\ University Hospital Bern, Bern, Switzerland
}

\section{Summary}

Objective: The objective is to report an easy and fast way of out-of-hospital induction of mild therapeutic hypothermia after prolonged cardiopulmonary resuscitation.

Method: Retrospective case report.

Case reports: We report two cases of witnessed outof-hospital cardiac arrest at an alpine ski resort. Both patients underwent prolonged cardiopulmonary resuscitation due to persistent ventricular fibrillation. On scene, mild therapeutic hypothermia was induced with packed snow either after return of spontaneous circulation or already during active resuscitation. For percutaneous coronary intervention, the patients were airlifted to a tertiary cardiology centre. During the 16 minute flight, they both reached the hypothermia target temperature of $33 \pm 1{ }^{\circ} \mathrm{C}$ and were kept hypothermic for 24 hours. After active rewarming and extubation, the patients recovered completely and did not suffer from any neurological dysfunction.

Discussion: We attribute the excellent outcome to the resolute implementation of the resuscitation and the early out-of-hospital onset of mild therapeutic hypothermia.

Key words: cardiopulmonary resuscitation (CPR); hypothermia; myocardial infarction; neurological dysfunction; helicopter emergency medical service (HEMS); snow

Funding/Financial Interest: None. internal vocational training at: Department of Intensive Care, Emergency Medicine and Anaesthesiology, Intensive Care Clinic, University Hospital Bern, Bern, Switzerland and fmi Hospital Interlaken, Institute of Anaesthesiology and Intensive Care, Unterseen, Switzerland in August 2008.
This series of case reports was presented as

\section{Introduction}

Background: Patients experiencing out-of-hospital cardiac arrests, have an extremely high mortality rate $[1,2]$. Cardiopulmonary resuscitation (CPR) of these patients generally has a poor outcome [3]. Even in cases of witnessed out-of-hospital cardiac arrests, the rate of discharge without neurological sequelae is extremely low [1-4]. If compared to historical control groups, mild therapeutic hypothermia following out-ofhospital cardiac arrest considerably improves cardiac and neurological outcome. At the same time, hypothermia rarely leads to uncontrollable adverse effects $[5,6]$. The early induction of hypothermia under active resuscitation seems highly beneficial and reduces the rate of anoxic brain damage even further [7, 8]. Despite an American Heart Association (AHA) and International Liaison Committee on Resuscitation (ILCOR) 2003 recommendation, the intra-hospital and even more so, the out-of-hospital implementation of therapeutic hypothermia remains poor [8-10, 13].

Objective: The objective is to report an easy and fast method of out-of-hospital induction of mild therapeutic hypothermia after prolonged cardiopulmonary resuscitation.

Methods and setting: Retrospective case report concerning two patients who were reanimated at an alpine ski resort in Switzerland. A HEMS-crew (helicopter emergency medical service) of the Swiss Air Rescue REGA airlifted the patients to the University Hospital Bern, Bern, Switzerland, where the percutaneous coronary interventions and the further treatment took place. Thereafter one of the patients was transferred to the intensive care unit (ICU) of the fmi Hospital In-

Correspondence:

Dr. Alexander J. Pfister

Universitätsspital Basel

Department of Anaesthesia

Spitalstrasse 21

CH-4031 Basel

apfister@uhbs.ch 
terlaken, Institute of Anaesthesiology and Intensive Care, Unterseen, Switzerland. Both patients expressed their written informed consent to undergo a scientific evaluation of their personal patient data.

\section{Case reports}

\section{Case 1}

A 72-year-old male skier experienced witnessed out-ofhospital cardiac arrest in a public restaurant (closed room, room temperature $18^{\circ} \mathrm{C}$ ) at the alpine ski resort of Grindelwald First, Switzerland. After collapsing (h), he underwent immediate bystander CPR. At $\mathrm{h}+4$ minutes, an automated external defibrillator (AED) was attached by basic life support and AED trained ski patrol first responders. The patient was resuscitated according to AHA and ILCOR 2005 guidelines. At the arrival of a HEMS team $(h+15)$, the patient was in persistent ventricular fibrillation, had already received two biphasic shocks, and presumably had aspirated gastric content. During the prolonged resuscitation period, he recurrently switched between ventricular fibrillation and pulseless electrical activity with accelerated idioventricular rhythm. At $\mathrm{h}+43$, the patient converted to normocardic sinus rhythm and experienced return of spontaneous circulation (ROSC). In total he received $3 \mathrm{mg}$ epinephrine, $450 \mathrm{mg}$ amiodarone and eight shocks (rectilinear biphasic ${ }^{\mathrm{TM}}$ waveform ZOLL ${ }^{\mathrm{TM}}$ AED Plus ${ }^{\mathbb{R}}$ ). The standard ECG-monitoring showed marked ST-segment elevations. After placing a nasogastric temperature probe (YSI ${ }^{\circledR}$ compensated oesophageal/rectal temperature probe, Data Sciences International $1^{\mathrm{TM}}$, range of operation $0^{\circ}$ to $60^{\circ} \mathrm{C}$, accuracy $\pm 0.2^{\circ} \mathrm{C}$ from $-1^{\circ}$ to $60^{\circ} \mathrm{C}$ and $\pm 0.1^{\circ} \mathrm{C}$ from $25^{\circ}$ to $45^{\circ} \mathrm{C}$, standard temperature probe used with Protocol Systems Inc. TM PROPAQ $106^{\circledR}$ EL patient monitoring system, routinely used for temperature detection under general anaesthesia), at approximately $55 \mathrm{~cm}$, therapeutic hypothermia was induced on scene. The upper thoracic aperture and the inguinal region where each packed with approximately $4 \mathrm{~kg}$ of fresh snow (see fig. 1). During the 16 minute airlift to the nearest tertiary cardiology centre, the patient's core temperature dropped from $35.8^{\circ} \mathrm{C}$ to $33.7^{\circ} \mathrm{C}$. At the cardiology centre, an anterolateral ST-elevation myocardial infarction (STEMI) with cardiogenic shock was diagnosed. Coronary angiography showed a onevessel disease. A proximal occlusion of the left anterior descending coronary artery was successfully recanalised (starting at $\mathrm{h}+98$ ). The left ventricular ejection fraction (LVEF) was
Figure 1

Packing of the upper thoracic aperture and the inguinal region each with approximately $4 \mathrm{~kg}$ of fresh snow (identical approach in Case 1 and 2).

The snow is modelled along the body on a large area, while pushed back underwear holds the packing together and increases surface pressure.

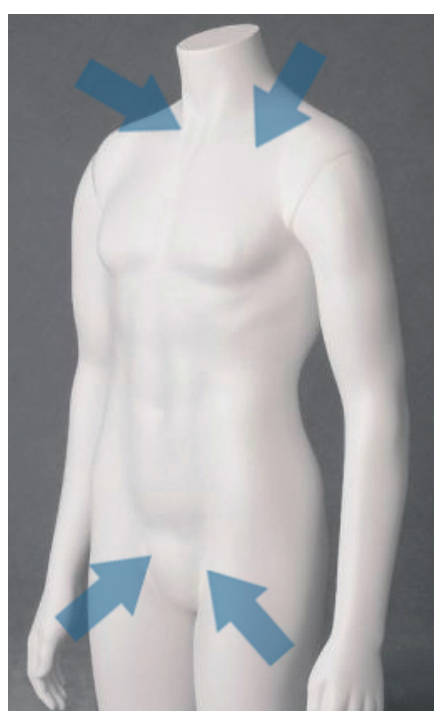

at $40 \%$. Subsequently, the patient was transferred to an ICU and kept in a hypothermia protocol $\left(33 \pm 1^{\circ} \mathrm{C}, \mathrm{MTRE}^{\mathrm{TM}}\right.$ Allon $^{\circledR}$ 2001 Control Unit, ThermoWrap ${ }^{\circledR}$, Mennen Medical Corp., USA) for 24 hours (see fig. 2). After active rewarming (0.5 ${ }^{\circ} \mathrm{C} / \mathrm{h}$ ) to $>36.0^{\circ} \mathrm{C}$ and extubation, he showed atrial fibrillation with rapid ventricular response and only suffered from mild anomia and atactic movements. The following day the patient developed massive aspiration pneumonia (Proteus mirabilis), as well as a ventilator-associated pneumonia (Pseudomonas sp.) with septic shock requiring inotropic support, reintubation and mechanical ventilation for a further five days. After transfer to a regular medical ward, the patient recovered completely without neurological deficiency and was discharged on day 26. Magnetic resonance imaging showed no signs of anoxic brain damage.

\section{Case 2}

Within six days, in the same ski resort, a 50-year-old male patient sustained witnessed out-of-hospital cardiac arrest whilst sledging (field temperature $+3^{\circ} \mathrm{C}$, overcast, slight snowfall). Bystander CPR started delayed at $\mathrm{h}+3$ minutes due to vigorous emesis. The same ski patrol first responders continued CPR $(\mathrm{h}+7)$ and delivered two biphasic shocks (same equipment) due to ventricular fibrillation. At the arrival of a HEMS team $(\mathrm{h}+14)$, the patient was in persistent ventricular fibrillation and had massively aspirated gastric content. Thera-

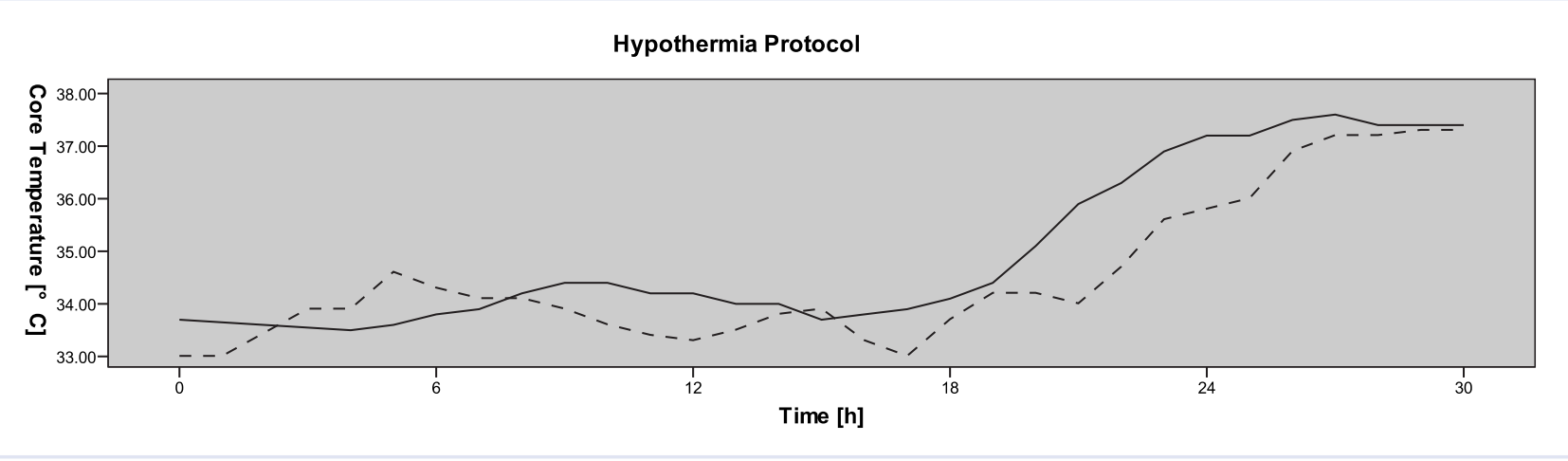

\section{Figure 2}

24-hour hypothermia protocol of both patients (Case 1 full line, Case 2 dashed line), starting at the time of admission to the emergency room of the tertiary cardiology centre $(h+88$ minutes for Case 1 and $h+67$ minutes for Case 2$)$. Core temperature measuring was done using either a pulmonary artery Swan-Ganz catheter or a nasogastric temperature probe. 
peutic hypothermia was induced with packed snow during resuscitation exactly in the way described in Case 1 (see fig. 1), whereas core temperature was not recorded under active resuscitation. The complete further treatment was similar to Case 1. The patient was in persistent ventricular fibrillation, until, at $\mathrm{h}+33$, he converted to bradycardic sinus rhythm experiencing ROSC. In total he received $5 \mathrm{mg}$ epinephrine, $300 \mathrm{mg}$ amiodarone and 13 shocks (monophasic ZOLL ${ }^{\text {тм }}$ PD $2000^{\circledR}$ ). For percutaneous coronary intervention, he was airlifted to the same cardiology centre in a 16 minute flight. On admission $(\mathrm{h}+67)$, his core temperature, measured by a nasogastric temperature probe (same type and manufacturer), had dropped to $33.0^{\circ} \mathrm{C}$. An inferolateral STEMI was diagnosed, and the patient was thereafter also kept in a hypothermia protocol (see fig. 2). Due to triage reasons, the coronary intervention started delayed at $\mathrm{h}+114$ minutes. Coronary angiography showed a 3 -vessel disease with a severely reduced LVEF of $30 \%$. An occlusion of a large second marginal branch was successfully recanalised, and the patient underwent staged interventions in the left anterior descending and the right coronary artery. Subsequently, he was transferred to an ICU and anew had to be reanimated due to ventricular fibrillation. After keeping him hypothermic for 24 hours (same hypothermia protocol, same cooling device), active rewarming and extubation, he was transferred to an intermediate care ward with complete motor aphasia and early aspiration pneumonia. Magnetic resonance imaging showed various older ischaemic lesions in the left hemisphere and cerebellum. Following this the patient recovered completely with no neurological deficiency and was discharged on day 19.

\section{Discussion}

The induction and continuation of mild therapeutic hypothermia strongly relies on correct temperature monitoring and the ability to achieve and maintain the intended temperature goal. However, during the induction of hypothermia, the main focus usually lies on reducing the time to achieve the temperature goal, i.e., a high cooling rate, whereas initial temperature control often seems to be neglected. Thus, fast surface cooling is per se associated with a higher rate of significant temperature overshoot [11]. Thus, uncontrolled and chaotic cooling of patients surviving out-of-hospital cardiac arrest increases temperature related arrhythmic episodes. Therefore, the induction of mild therapeutic hypothermia should only be considered, if reliable temperature monitoring is available on scene.

The reliability and accuracy of the many electrophysical ways of temperature detection (thermocouples, thermistors, resistance temperature detectors, pyrometers, infrared detectors) and application of thermal probes (cutaneous, oral, nasal, nasopharyngeal, nasogastric, gastric, deep rectal, bladder temperature, intravenous, intra-arterial) remains a constant topic of academic controversy and will not be further discussed here. In contrast, during practical application and everyday use of temperature probes, the question of accuracy hardly ever arises, as no redundant measuring is performed or available. In the past, the YSI ${ }^{\circledR}$ compen- sated oesophageal/rectal temperature probe, as used with the two patients, has shown good practical field reliability with avalanche victims. Furthermore, during US Air Force testing, its reliability during the airborne use (altitude change, temperature change, humidity change) was only questioned as regards electromagnetic interference testing [12]. It may thus be assumed that the applied prehospital method of core temperature measuring is comparable to data collected during intra-hospital induction of mild therapeutic hypothermia.

Currently, the most common way, to reach the intended hypothermia target temperature $\left(33 \pm 1^{\circ} \mathrm{C}\right)$, is the rapid infusion of $4{ }^{\circ} \mathrm{C}$ crystalloids, taking up to 75 minutes [14]. However, consequent induction and temperature control is hardly possible in the emergency room and catheterization laboratory setting. Therefore, the core temperature reaches the target value at earliest with a systematic delay of 1 to 16 hours [5, 6, 14-16]. Out-of-hospital induction of mild therapeutic hypothermia is hardly ever done, especially when short transportation times are anticipated [13]. If induced on scene by cool packs, target temperature is commonly reached within 2 to 3 hours [17]. In contrast, with our snow-packed patients, target temperature was already reached within the 16 -minute airlift. Whether the meteorological conditions affected the cooling rate remains unknown. Case 1 underwent indoor CPR in a heated place, while Case 2 was already found in the snow. Nevertheless, both patients had similar cooling rates and both reached the target temperature within the same time.

Out of hospital, there are several advantages of snow over ice or the application of glycerol or encapsulated saline solution containing cool packs. In the alpine region, snow is omnipresent during most of the year. Snow hardly ever causes more than grade 1 frostbites (both patients indeed sustained grade 1 frostbites). Modelling snow along the body allows full surface contact on a large area (pushed back underwear holds the snow packing together and increases surface pressure). Melting snow has an enormous entropic effect and furthermore, unlike ice, the amount of snow used with our patients melts within 20 minutes and will not cause further harm.

We attribute the excellent outcome to the resolute implementation of the resuscitation and the early outof-hospital onset of hypothermia as well as to its rapid induction. Whilst these two reanimations may seem anecdotal, the positive outcome encourages us to recommend snow packing in similar cases.

We would like to thank the dedicated ski patrol first responders of the Grindelwald First ski resort, Switzerland, for their unequalled motivation to advanced vocational training and their excellent performance during these two reanimations. 


\section{References}

1 Davis DP, Fisher R, Aguilar S, Metz M, Ochs G, McCallum-Brown L, et al. The feasibility of a regional cardiac arrest receiving system. Resuscitation. 2007;74(1):44-51.

2 Dunne RB, Compton S, Zalenski RJ, Swor R, Welch R, Bock BF. Outcomes from out-of-hospital cardiac arrest in Detroit. Resuscitation. 2007;72(1):59-65.

3 Horsted TI, Rasmussen LS, Meyhoff CS, Nielsen SL. Long-term prognosis after out-of-hospital cardiac arrest. Resuscitation. 2007;72(2): 214-8.

4 Eckstein M, Stratton SJ, Chan LS. Cardiac Arrest Resuscitation Evaluation in Los Angeles: CARE-LA. Ann Emerg Med. 2005;45(5):504-9.

5 Bernard SA, Gray TW, Buist MD, Jones BM, Silvester W, Gutteridge G, Smith K. Treatment of comatose survivors of out-of-hospital cardiac arrest with induced hypothermia. N Engl J Med. 2002;346(8):557-63.

6 Mild therapeutic hypothermia to improve the neurologic outcome after cardiac arrest. N Engl J Med. 2002;346(8):549-56.

7 Boddicker KA, Zhang Y, Zimmerman MB, Davies LR, Kerber RE. Hypothermia improves defibrillation success and resuscitation outcomes from ventricular fibrillation. Circulation. 2005;111(24):3195-201.

8 Soar J, Nolan JP. Mild hypothermia for post cardiac arrest syndrome. Bmj. 2007;335(7618):459-60

9 Abella BS, Rhee JW, Huang KN, Vanden Hoek TL, Becker LB. Induced hypothermia is underused after resuscitation from cardiac arrest: a current practice survey. Resuscitation. 2005;64(2):181-6.

10 Nolan JP, Morley PT, Vanden Hoek TL, Hickey RW, Kloeck WG, Billi J, et al. Therapeutic hypothermia after cardiac arrest: an advisory statement by the advanced life support task force of the International Liaison Committee on Resuscitation. Circulation. 2003;108(1):118-21.
11 Flint AC, Hemphill JC, Bonovich DC. Therapeutic hypothermia after cardiac arrest: performance characteristics and safety of surface cooling with or without endovascular cooling. Neurocrit Care. 2007;7(2): 109-18.

12 Suffoletto BP, Salcido DD, Menegazzi JJ. Use of Prehospital-Induced Hypothermia After Out-of-Hospital Cardiac Arrest: A Survey of the National Association of Emergency Medical Services Physicians. Prehosp Emerg Care. 2008;12(1):52-6.

13 Edman KA. AL/CF-TR-1996-0118: Testing and Evaluation of the Protocol Systems Propaq 106EL Physiologic Patient Monitor. United States Air Force, Armstrong Laboratory (AFMC), Crew Systems Directorate, Crew Technology Division, Systems Research Branch, Brooks, Texas, USA. 1996. http://www.dtic.mil/cgibin/GetTRDoc?AD= ADA317189\&Location $=$ U2\&doc=GetTRDoc.pdf

14 Bernard SA, Rosalion A. Therapeutic hypothermia induced during cardiopulmonary resuscitation using large-volume, ice-cold intravenous fluid. Resuscitation. 2008;76(2): 311-3.

15 Oddo M, Schaller MD, Feihl F, Ribordy V, Liaudet L. From evidence to clinical practice: effective implementation of therapeutic hypothermia to improve patient outcome after cardiac arrest. Crit Care Med. 2006; 34(7):1865-73.

16 Froehler MT, Geocadin RG. Hypothermia for neuroprotection after cardiac arrest: mechanisms, clinical trials and patient care. J Neurol Sci. 2007;261(1-2):118-26.

17 Belliard G, Catez E, Charron C, Caille V, Aegerter P, Dubourg O, et al. Efficacy of therapeutic hypothermia after out-of-hospital cardiac arrest due to ventricular fibrillation. Resuscitation. 2007;75(2):252-9. 